Proceedings of the

International Geometry Center

Vol. 10, no. 2 (2017) pp. 1-7

\title{
On colorings and isometries
}

\author{
Igor Protasov
}

\begin{abstract}
In the first section we prove some isometric versions of the classical Ramsey theorem. In the second section we discuss open problems on metrically Ramsey ultrafilters. Given a metric space $(X, d)$, we say that a mapping $\chi:[X]^{2} \rightarrow\{0.1\}$ is an isometric coloring if $d(x, y)=d(z, t)$ implies $\chi(\{x, y\})=\chi(\{z, t\})$, where $[X]^{2}$ is the set of all two-element subsets of $X$. A free ultrafilter $\mathcal{U}$ on an infinite metric space $(X, d)$ is called metrically Ramsey if, for every isometric coloring $\chi$ of $[X]^{2}$, there is a member $U \in \mathcal{U}$ such that the set $[U]^{2}$ is $\chi$-monochrome.
\end{abstract}

\section{ISOMETRIC VERSIONS OF RAMSEY THEOREM}

Motivation and results. For any natural numbers $n, r$, there exists a natural number $m$, such that for any $r$-coloring of edges of the complete graph $K_{m}$, there is a monochrome copy of $K_{n}$.

This elegant statement is a graph version of Ramsey theorem, one of the milestones of Ramsey Theory. For history (with exposition of the original paper of Frank Ramsey) and foundations of this branch of Combinatorics, see [1]. For geometrical aspects, in particular, chromatic numbers of $\mathbb{R}^{n}$, see [8].

Clearly, $K_{n}$ contains an isomorphic copy of any graph with $\leq n$ vertices, so for every finite graph $\Gamma$ and a natural number $r$, there exists a natural number $m$ such that, for any $r$-coloring of edges of $K_{m}$, there is a monochrome copy $\Gamma^{\prime}$ of $\Gamma$. But this $\Gamma^{\prime}$, as a rule, lies in $K_{m}$ very non-isometrically with respect to $\Gamma$. We need some definitions to explain this passage.

All graphs are supposed to be finite and connected. Every graph $\Gamma$ with the set of vertices $V(\Gamma)$ and the set of edges $E(\Gamma)$ (each edges in an unordered pair $\{u, v\}$ of two distinct vertices) can be considered as the metric space $\left(V(\Gamma), d_{\Gamma}\right)$ with the path metric $d_{\Gamma}$ defined by the rule: $d(u, v)$ is the length (by edges) of a shortest path (called geodesic) between $u$ and

2010 Mathematics Subject Classification: 51K05, 05D10

Keywords: isometric coloring, isometric Ramsey theorem, metrically Ramsey ultrafilters 
$v$. Given two graphs $\Gamma$ and $G$, a mapping $f: V(\Gamma) \rightarrow V(G)$ is an isometric embedding if, for any $u, v \in V(\Gamma), d_{\Gamma}(u, v)=d_{G}(f(u), f(v))$. Thus, $\Gamma$ is isometrically embedded into $K_{m}$ if and only if $\Gamma$ is complete.

Now we take an arbitrary graph $\Gamma$, natural number $r$ and ask if there exists a graph $G$ such that, for every $r$-coloring of $E(G)$, there is a monochrome isometric copy of $\Gamma$ ? The answer is positive and follows from Theorem 1.3 in [4]. But the construction of that $G$ in [4] essentially depends on $\Gamma$ and could be very complicated.

We show that the choice of $G$ is very simple if $\Gamma$ is isometrically embeddable into the Cartesian product of complete graphs.

For some characterization of graphs isometrically embeddable into $K_{n}^{m}$ see [9].

Theorem 1.1. Assume that a graph $\Gamma$ is isometrically embedded into $K_{n}^{m}$, and let $r$ be a natural number. Then there exists a natural number $N$ such that, for $G=K_{N}^{m r-r+1}$ and any $r$-coloring of $V(G)$ and $r$-coloring of $E(G)$, the graph $G$ contains an isometrically embedded, vertex-monochrome, and edge-monochrome copy of $\Gamma$.

We recall that the Cartesian product $G=G_{1} \times \ldots \times G_{n}$ of graphs $G_{1}, \ldots, G_{n}$ is a graph with the set of vertices

$$
V(G)=V\left(G_{1}\right) \times \ldots \times V\left(G_{n}\right)
$$

and the set of edges

$$
E(G)=\bigcup_{i \leq n} E_{i}(G)
$$

defined by the rule: for $u=\left(u_{1}, \ldots, u_{n}\right)$ and $v=\left(v_{1}, \ldots, v_{n}\right)$, $u v \in E_{i}(G) \Longleftrightarrow u_{i} v_{i} \in E\left(G_{i}\right), \quad u_{k}=v_{k}, \quad k \in\{1, \ldots, i-1, i+1, \ldots, n\}$.

By $K_{n}^{m}$, we denote the Cartesian product of $m$ complete graphs $K_{n}$.

If $S_{1}, \ldots, S_{n}$ are subgraphs of $G_{1}, \ldots, G_{n}$, we say that $S_{1} \times \ldots \times S_{n}$ is a box in $G$. We say that a graph $H_{1} \times \ldots \times H_{k}$ is box embeddable into $G$ if, after some rearrangement of $\{1, \ldots, n\}$, there is a box

$$
S_{1} \times \ldots \times S_{k} \times\left\{u_{k+1}\right\} \times \ldots \times\left\{u_{n}\right\}
$$

in $G$ such that $S_{i} \simeq H_{i}, i \in\{1, \ldots, k\}$, where the sign $\simeq$ means an isomorphism.

We extract Theorem 1.1 from the following statements announced in [6].

Theorem 1.2. For any natural numbers $n, m, r$ there exists a natural number $N$ such that for every $r$-coloring of $V\left(K_{N}^{m}\right)$ the graph $K_{N}^{m}$ contains a monochrome box copy of $K_{n}^{m}$. 
Theorem 1.3. For any natural numbers $n, m, r$ there exists a natural number $N$ such that, for every $r$-coloring of $E\left(K_{N}^{m r-r+1}\right)$ the graph $K_{N}^{m r-r+1}$ contains a monochrome box copy of $K_{n}^{m}$.

If $G_{1}, \ldots, G_{n}$ are directed graphs, we consider $G=G_{1} \times \ldots \times G_{n}$ as a graph endowed with the unique orientation $\mathcal{O}$ such that the restriction of $\mathcal{O}$ to each $E_{i}(G)$ coincides with orientation of $E\left(G_{i}\right)$.

Theorem 1.4. Let $n, m$ be natural numbers and let $G_{1}, \ldots, G_{m}$ be acyclically oriented copies of $K_{n}$. Then there exists a natural number $N$ such that for any acyclic orientation of $K_{n}^{m}$ there is a directed box copy of $G_{1}, \ldots, G_{m}$ in $K_{N}^{m}$.

\section{Proofs}

Theorem 1.2 is a simple corollary of Lemma 1 from [2].

We will extract Theorem 1.3 from some more general result.

Say that an edge-coloring $\chi$ of $G_{1} \times \ldots \times G_{n}$ is a face coloring if the restrictions of $\chi$ to each $E_{i}\left(G_{1} \times \ldots \times G_{n}\right)$ is monochrome.

Theorem 1.5. For any natural numbers $n, k, r$ there exists a natural number $N$ such that for $G=K_{N}^{k}$ and any $r$-coloring $\chi$ of $E(G)$ the restriction of $\chi$ to some box $G_{1} \times \ldots \times G_{k}, G_{i} \simeq K_{n}$ is a face coloring.

Proof. Let us fix $n, r$ and proceed on induction by $k$. For $k=1$, we have the Ramsey theorem.

The transition from $k$ to $k+1$ will be done in five steps.

Step 1. At first we use Theorem 1.2 to choose $p$ such that, for $P=K_{p}^{k}$ and any $r$-coloring of $V(P)$, there is a monochrome box $P_{1} \times \ldots \times P_{k}$, $P_{i} \simeq K_{n}$ in $P$.

Now apply the inductive assumption to choose $q$ such that, for $Q=K_{q}^{k}$ and any $r$-coloring $\Psi$ of $E(Q)$, the restriction of $\Psi$ to some box $Q_{1} \times \ldots \times Q_{k}$, $Q_{i} \simeq K_{p}$ in $Q$ is a face coloring.

Step 2. Given a natural number $t$, we put $s(t)=t r^{|E(Q)|}$ and note that, by the pigeonhole principle, for every $r$-coloring $\Phi$ of $E\left(Q \times K_{s(t)}\right)$ there is a subset $T \subset V\left(K_{s(t)}\right)$ such that $|T|=t$ and all restrictions of $\Phi$ to $E(Q \times\{t\}), t \in T$ coincide.

Step 3. We define the iterated Ramsey numbers $R^{(i)}(n, r)$ by

$$
R^{1}(n, r)=R(n, r), \quad R^{(i+1)}(n, r)=R\left(R^{(i)}(n, r), r\right),
$$

where $R(l, r)$ is the minimal natural number such that for any $r$-coloring of $E\left(K_{R(l, r)}\right)$ there is a monochrome copy of $K_{l}$. 
Step 4. We put $t=R^{(|V(P)|)}(n, r)$, choose $N$ such that $N>s(t), N>q$, and take an arbitrary $r$-coloring $\chi$ of $E\left(K_{N}^{k} \times K_{N}\right)$.

Regard $Q$ as a box of $K_{N}^{k}$. Then it follows from the definition of $s(t)$, there exists a subset $X$ of $V\left(K_{N}\right)$ such that $|X|=t$ and all restrictions of $\chi$ to $E(Q \times\{x\}), x \in X$, coincide. Then by the choice of $Q$, each restrictions of $\chi$ to $E(P \times\{x\})$, with $P=Q_{1} \times \ldots \times Q_{k}$ and $x \in X$, is a face coloring.

Step 5. Enumerate $V(P)=\left\{u_{1}, \ldots, u_{|V(P)|}\right\}$ and use Step 4 to choose subsets $X_{1}, \ldots, X_{|V(P)|}$ of $X$ such that $X_{1} \supset \ldots \supset X_{|V(P)|},\left|X_{|V(P)|}\right|=n$, and each $E\left(\left\{u_{i}\right\} \times Y_{i}\right)$ is $\chi$-monochrome, where $Y_{i}$ is a complete graph with the set of vertices $X_{i}$.

By the choice of $P$, there exists a box $P_{1} \times \ldots \times P_{k}$ in $P$ such that $P_{i} \simeq K_{n}$ and all $E\left(\{u\} \times Y_{|V(P)|}\right), u \in V\left(P_{1} \times \ldots \times P_{k}\right)$, are of the same color.

Hence, $\chi$ is a face coloring on $P_{1} \times \ldots \times P_{k} \times Y_{|V(P)|}$.

To get Theorem 1.3, we apply Theorem 1.5 with $k=(m-1) r+1$.

Proof of Theorem 1.4. We fix $n$ and proceed on induction by $m$. For $m=1$, the statement is evident because each acyclic orientation of a complete graph is uniquely determined by some ordering of its vertices.

We make the transition from $m$ to $m+1$ in four steps.

Step 1. Use Theorem 1.2 to choose $p$ such that for $P=K_{p}^{m}$ and any $n$ !-coloring of $V(P)$ there exists a monochrome box $P_{1} \times \ldots \times P_{m}$ in $P$ with $P_{i} \simeq K_{n}$.

Now we use the inductive assumption to choose $q$ such that for $Q=K_{q}^{m}$ and any acyclic orientation $\Psi$ of $E(Q)$ the restriction of $\Psi$ to some box $Q_{1} \times \ldots \times Q_{m}$ with $Q_{i} \simeq K_{p}$ is induced by orientations of $E\left(Q_{1}\right), \ldots, E\left(Q_{m}\right)$.

Step 2. Put $s(n)=n 2^{|E(Q)|}$ and choose $N$ so that $N>s(n)$ and $N>q$. We also take an arbitrary acyclic orientation $\mathcal{O}$ of $K_{N}^{m} \times K_{N}$.

Step 3. Regard $Q$ as a box of $K_{N}^{m}$. By the definition of $s(n)$, there exists a subset $X$ of $V\left(K_{N}\right)$ such that $|X|=n$ and all restrictions of $\mathcal{O}$ to $E(Q \times\{x\}), x \in X$, coincide. By the choice of $Q$, each restriction $\chi$ to $E(P \times\{x\})$, with $P=Q_{1} \times \ldots \times Q_{m}$ and $x \in X$, is induced by orientations of $E\left(Q_{1}\right), \ldots, E\left(Q_{m}\right)$.

Step 4. Denote by $Y$ the complete graph on the set of vertices $X$. Since there are $n$ ! acyclic orientations of $Y$, it follows from the choice of $P$ that there exists a box $P_{1} \times \ldots \times P_{m}$ in $P$ such that $P_{i} \simeq K_{n}$ and all $\{u\} \times Y$, $u \in P_{1} \times \ldots \times P_{m}$, have the same type of orientation: $\{u\} \times v w \in \mathcal{O}$ if and only if $\left\{u^{\prime}\right\} \times v w \in \mathcal{O}$.

Hence, $P_{1} \times \ldots \times P_{m} \times Y$ is the desired box in $K_{N}^{m+1}$. 


\section{Metrically Ramsey ultrafilters}

We recall that a family $\mathfrak{F}$ of subsets of a set $X$ is a filter if $X \in \mathfrak{F}$ and $A, B \in \mathfrak{F}, B \subseteq C$ imply $A \bigcap B \in \mathfrak{F}, C \in \mathfrak{F}$. A filter being maximal by inclusion is called an ultrafilter. An ultrafilter $\mathcal{U}$ is free if $\bigcap \mathcal{U}=\emptyset$.

Let $X$ be an infinite set and let $\mathfrak{F}$ be some family of $\{0,1\}$-colorings of the set $[X]^{2}$ of all two-elements subsets of $X$. We say that a free ultrafilter $\mathcal{U}$ on $X$ is Ramsey with respect to $\mathfrak{F}$ if for any coloring $\chi \in \mathfrak{F}$ there exists $U \in \mathcal{U}$ such that $[U]^{2}$ is $\chi$-monochrome. In the case in which $\mathfrak{F}$ is the family of all $\{0,1\}$-colorings of $[X]^{2}$ we get the classical definition of Ramsey ultrafilters. It is well-known that $\mathcal{U}$ is a Ramsey ultrafilter if and only if $\mathcal{U}$ is selective, i.e. for every partition $\mathcal{P}$ of $X$ either $P \in \mathcal{U}$ for some $P \in \mathcal{P}$ or there exists $U \in \mathcal{U}$ such that $|U \cap P| \leq 1$ for each $P \in \mathcal{P}$.

Given a metric space $(X, d)$, we say that a mapping $\chi:[X]^{2} \rightarrow\{0,1\}$ is an isometric coloring if $d(x, y)=d(z, t)$ implies $\chi(\{x, y\})=\chi(\{z, t\})$. We note that every isometric coloring $\chi$ is uniquely determined by some mapping

$$
f: d(X, X) \backslash\{0\} \rightarrow\{0,1\} .
$$

Indeed, take an arbitrary $r \in d(X, X) \backslash\{0\}$ and choose $\{x, y\} \in[X]^{2}$ such that $d(x, y)=r$, and put $f(r)=\chi(\{x, y\})$. Conversely, given a map $f: d(X, X) \backslash\{0\} \rightarrow\{0,1\}$, we define $\chi$ by $\chi(\{x, y\})=f(d(x, y))$.

We say that a free ultrafilter on an infinite metric space $(X, d)$ is metrically Ramsey if $\mathcal{U}$ is Ramsey with respect to all isometric colorings of $[X]^{2}$.

Let $G$ be a group and let $X$ be a $G$-space with an action

$$
G \times X \rightarrow X, \quad(g, x) \mapsto g x .
$$

A coloring $\chi:[X]^{2} \rightarrow\{0.1\}$ is called $G$-invariant if $\chi(\{x, y\})=\chi(\{g x, g y\})$ for all $\{x, y\} \in[X]^{2}$ and $g \in G$. A free ultrafilter $\mathcal{U}$ on $X$ is called $G$-Ramsey if $\mathcal{U}$ is Ramsey with respect to the family of all $G$-invariant colorings of $[X]^{2}$.

We consider the following special case: $X$ is a metric space and $G$ is a group of isometries of $X$. Clearly, every isometric coloring of $[X]^{2}$ is $G$ invariant. If $G$ is metrically 2 -transitive (if $d(x, y)=d(z, t)$ then there is $g \in G$ such that $g\{x, y\}=\{z, t\})$, then every $G$-invariant coloring of $[X]^{2}$ is an isometric coloring.

We take the group $\mathbb{Z}$ of integers, put $X=\mathbb{Z}$ and consider the action $\mathbb{Z}$ on $X$ by $(g, x)=g+x$.

- Is each $\mathbb{Z}$-Ramsey ultrafilter selective?

This question appeared in [5] and, to our knowledge, remains open. We endow $\mathbb{Z}$ with the metric $d(x, y)=|x-y|$. By above paragraph an ultrafilter $\mathcal{U}$ on $\mathbb{Z}$ is $\mathbb{Z}$-Ramsey if and only if $\mathcal{U}$ is metrically Ramsey. 
- Is each metrically Ramsey ultrafilter on $\mathbb{Z}$-selective?

This is an equivalent form of the above question. The case of $\mathbb{Z}$ is evidently equivalent to the case of $\mathbb{N}$.

Some partial results are obtained in [5], [7].

Theorem 2.1. Let $\mathcal{U}$ be a metrically Ramsey ultrafilter on $\mathbb{N}$ and $f: \mathbb{N} \rightarrow \mathbb{N}$ be a mapping such that $f(x)>x$ for each $x \in \mathbb{N}$. Then there exists a member $U \in \mathcal{U}$ having no subsets of the form $\{a, a+x, a+f(x)\}$. In particular, for $f(x)=2 x$, some member of $\mathcal{U}$ has no arithmetic progressions of length 2.

We say that a subset $T=\left\{t_{n}: t_{n}<t_{n+1}, n<\omega\right\}$ of $\mathbb{N}$ is thin if $\left(t_{n+1}-t_{n}\right) \rightarrow \infty$ as $n \rightarrow \infty$.

The following theorem is Corollary 2 from [5].

Theorem 2.2. Every metrically Ramsey ultrafilter $\mathcal{U}$ on $\mathbb{N}$ has a member with no subsets of the form $\{x, y, x+y\}, x \neq y$.

Theorem 2.3. If a metrically Ramsey ultrafilter $\mathcal{U}$ on $\mathbb{N}$ has a thin subset $T \in \mathcal{U}$ then there exists a mapping $\varphi: \mathbb{N} \rightarrow \omega$ such that the ultrafilter $\varphi(\mathcal{U})$ is selective and $\varphi$ is finite-to-one on some member $U \in \mathcal{U}$.

Surprisingly or not, the case of ultrametric spaces is cardinally different and much more easy to explore. By [7] every infinite ultrametric space $X$ has a countable subset $Y$ such that any ultrafilter $\mathcal{U}$ on $X$ satisfying $Y \in \mathcal{U}$ is metrically Ramsey.

In connection with Theorem 2.3, we ask

Question 2.4. Let $\mathcal{U}$ be a metrically Ramsey ultrafilter on $\mathbb{N}$. Does there exist a thin subset $U \in \mathcal{U}$ ?

Question 2.5. Assume that a metrically Ramsey ultrafilter $\mathcal{U}$ on $\mathbb{N}$ has a thin member. Is $\mathcal{U}$ selective?

By $\left[3\right.$, Theorem 6.2], there is a coloring $\chi:[\mathbb{R}]^{2} \rightarrow\{0,1\}$ such that if $X \subset \mathbb{R}$ and $[X]^{2}$ is $\chi$-monochrome then $|X| \leq \omega$.

We endow $\mathbb{R}$ with the natural metric $d(x, y)=|x-y|$ and ask

Question 2.6. Does there exist an isometric coloring $\chi:[\mathbb{R}]^{2} \rightarrow\{0,1\}$ such that if $[X]^{2}$ is monochrome then $|X| \leq \omega$ ?

We endow the Cantor cube $\{0,1\}^{\omega}$ with the standard metric and ask 
Question 2.7. Does there exist an isometric coloring

$$
\chi:\left[\{0,1\}^{\omega}\right]^{2} \rightarrow\{0,1\}
$$

such that if $[X]^{2}$ is monochrome then $|X| \leq \omega$ ?

\section{REREFENCES}

[1] Ronald L. Graham, Bruce L. Rothschild, Joel H. Spencer. Ramsey theory. John Wiley \& Sons, Inc., New York, 1980. Wiley-Interscience Series in Discrete Mathematics, A Wiley-Interscience Publication.

[2] A. W. Hales, R. I. Jewett. Regularity and positional games. Trans. Amer. Math. Soc., 106:222-229, 1963.

[3] Kenneth Kunen. Combinatorics. In Handbook of mathematical logic, volume 90, pages 371-401. North-Holland Publishing Co., Amsterdam-New York-Oxford, 1977.

[4] Jaroslav Nešetřil, Vojtěch Rödl. Sparse Ramsey graphs. Combinatorica, 4(1):71-78, 1984.

[5] Oleksandr Petrenko, Igor Protasov. Selective and Ramsey ultrafilters on G-spaces. Notre Dame Journal of Formal Logic, 58(3):453-459, 2017.

[6] I. Protasov. Isometric versions of ramsey theorem. Eureka. A Journal of The Archimedeans, to appear.

[7] I. Protasov, K. Protasova. Metrically Ramsey ultrafilters. arXiv:1704.07824, 2017.

[8] Alexander Soifer, editor. Ramsey theory, volume 285 of Progress in Mathematics. Birkhäuser/Springer, New York, 2011. Yesterday, today, and tomorrow, Papers from the workshop held at Rutgers University, Piscataway, NJ, May 27-29, 2009.

[9] Peter M. Winkler. Isometric embedding in products of complete graphs. Discrete Applied Mathematics, 7(2):221-225, Feb 1984.

Received: May 5, 2017, accepted: July 25, 2017.

Igor Protasov

Faculty of Computer Science and Cybernetics, Taras Shevchenko National University of Kyiv, Academic Glushkov ave. 4D, 03680 Kyiv, Ukraine

Email: i.v.protasov@gmail.com 